\title{
A case of HPV-53-related cervical cancer in an elderly patient
}

This article was published in the following Dove Press journal:

Clinical Interventions in Aging

II November 2014

Number of times this article has been viewed

\section{Marusya Lieveld' \\ Elizaveta Padalko 2,3 \\ Marleen Praet ${ }^{4}$ \\ Davy Vanden Broeck'}

'Department of Uro/gynaecology, Ghent University Hospital, Ghent, Belgium; '2Department of Clinical Chemistry, Microbiology and Immunology, Ghent University Hospital, Ghent, Belgium;

${ }^{3}$ School of Life Sciences, Hasselt University, Diepenbeek, Belgium; ${ }^{4} \mathrm{~N}$. Goormaghtigh Institute of Pathology, Ghent University Hospital, Ghent, Belgium
Correspondence: Marusya Lieveld The International Centre for Reproductive Health - Ghent University, De Pintelaan I85 UZPI I4,

9000 Ghent, Belgium

Tel +3293323564

Email marusya.lieveld@ugent.be

\section{Dear editor}

Zappacosta et al ${ }^{1}$ recently published a case report concerning a human papillomavirus (HPV)-positive invasive cervical cancer in a 79-year-old women who had a history of normal Pap smears. In this article, Anyplex II HPV28 (Seegene) is used for HPV genotyping of formalin-fixed paraffin embedded (FFPE) tissue, liquid based cytology (LBC) specimens and urine samples. It is suggested that HPV53 is present exclusively in the cervical cancer cells, lymph node metastases, and atypical urinary cells of one single case while the surrounding CIN2+ tissue revealed ten different HPV strains. Unfortunately, the HPV genotype results for lymph nodes and urinary cells are not presented while these results underline the potential role of HPV53 in oncogenesis. Moreover, it is generally accepted that one lesion is caused by one HPV infection, detection of multiple HPV types thus indicates the presence of multiple infections, ${ }^{2}$ suggesting that this patient may have several lesions.

In Figure 3 in Zappacosta et $\mathrm{al}^{1}$ two melting peaks (-d(RFU)/dt) for HPV53 are displayed, one detected by probe A in plasmid DNA (referred to as a positive internal control), and one detected by probe B in cervical cancer cells. However, Anyplex II HPV28 only uses one target specific primer/catcher/pitcher set for each HPV type. The internal positive control used in Anyplex II HPV28 is human $\beta$-globin and not plasmid DNA as indicated by Zappacosta et al. ${ }^{1}$ It is included to monitor whether the sample is adequate for analysis and to exclude polymerase chain reaction (PCR)-inhibition which may lead to false negative results. The test also includes external positive controls which consist of HPV type-specific plasmids, in order to validate whether the prepared master mix accurately detects the indicated HPV types. Nevertheless, the authors could opt to use synthesized plasmid HPV53 DNA as an extra internal positive control by adding it to the sample but this could not be derived from the article. In either case, melting curve analysis of the positive control will give a melting profile consisting of melting curves specific for each HPV type included in the positive control (personal communication Seegene). Because the shape of the melting curve and the position of the peak is a signature for each HPV type, infection with a particular HPV type will give rise to a particular melting curve. Therefore, it is expected that HPV53 infection in cervical cancer cells (right melting curve in Figure 3, Zappacosta et al ${ }^{1}$ ) will give the exact same melting profile as HPV53 observed in the positive control (left melting curve in Figure 3, Zappacosta et $\mathrm{al}^{1}$ ). This is not the case, as it can be deduced from Figure 3, Zappacosta et al. The melting peak of HPV53 in the positive control differs approximately $8^{\circ} \mathrm{C}$ from the HPV type detected in cervical cancer cells. 
The Anyplex II HPV28 is based on melting curve analysis using the TOCETM technology. In contrast to conventional High Resolution Melt (HRM) analysis, the TOCETM technology enables identification of multiple targets (in this case, HPV types) simultaneously in a single channel by controlling the catcher melting temperature (catcher-Tm). The catcher is an artificial fluorescently labeled template; it is designed in such a way that the tagging portion of "the pitcher", which previously recognized and hybridized with the target sequence, hybridizes with the catcher. Because each HPV-specific pitcher will hybridize with a catcher of a distinctive length causing a temperature shift during the melting out, different HPV types will be detected in one single channel using one fluorophore. Hereupon, catchers are labeled with different fluorophores (FAM, HEX, Cal Red 610, Quasar 670, Quasar 705) which are detected using different channels allowing multiplexing of 28 different HPV genotypes. Anyplex II HPV28 detects and differentiates 19 high-risk (16, 18, 26, 31, 33, 35, 39, 45, 51, 52, 53, 56, 58, 59, 66, 68, $69,73,82)$ and 9 low-risk HPV genotypes $(6,11,40,42,43$, 44, 54, 61, 70). The kit consists of two independent panels: Panel A with 14 high-risks (16, 18, 31, 33, 35, 39, 45, 51, $52,56,58,59,66,68)$ and Panel B with 5 high-risks $(26,53$, $69,73,82$ ) and 9 low-risks HPV. ${ }^{3-5}$

Zappacosta et al described in their article that urine samples were analyzed using Hybrid capture II HPV test (HC2 HPV test). ${ }^{1}$ To our knowledge, HC2 HPV test has not yet been optimized for urine samples. ${ }^{6,7}$ In-house optimization of urine samples for HC2 HPV could have been performed, but this is not specified in the article. It is also unclear why the authors carried out the HC2 HPV test since the results generated by Anyplex II HPV28 and HC2 are not compared with each other in the Results section. Moreover, Anyplex II HPV28 detects all of the genotypes included in HC2, which makes the potential complementary use of HC2 redundant.

To prevent sample-to-sample contamination, the microtome blade and working space were cleaned with DNA
$\operatorname{Away}^{\text {TM }}$ (Thermo Fischer Scientific) after sectioning of each FFPE test sample. However, it is unclear whether a negative control was included in the experiments in order to exclude cross-contamination. Validation for cross-contamination could be performed beforehand, but this is not reported in the article. A negative control (a tissue that is shown negative for the tested HPV types) should be cut between the test samples ideally after a positive specimen to verify absence of cross-contamination.

In conclusion, based on the observation that the melting curve of the HPV type detected in the cervical cancer cells does not correspond with the melting curve for HPV53 plasmid DNA, it can be deduced that another HPV type might be involved in the cervical cancer cells. Hence, it cannot be concluded that HPV53 is solely responsible in oncogenesis in this case as previously suggested by Zappacosta et al. ${ }^{1}$

\section{Disclosure}

All authors state that they have no conflicts of interest in this work.

\section{References}

1. Zappacosta R, Lattanzio G, Viola P, et al. A very rare case of HPV-53related cervical cancer, in a 79-year-old woman with a previous history of negative Pap cytology. Clin Interv Aging. 2014;9:683-688.

2. Quint W, Jenkins D, Molijn A, et al. One virus, one lesion - individual components of CIN lesions contain a specific HPV type. J Pathol. 2012; 227(1):62-71.

3. Seegene.com. Available from: http://www.seegene.com/en/research/ core_040.php. Accessed July 7, 2014.

4. Seegene.com. Available from: http://seegene.com/en/any/HPV28_010.php. Accessed July 7, 2014.

5. Seegene.com. Available from: http://seegene.com/en/any/HPV28_030.php. Accessed July 7, 2014.

6. Vorsters A, Micalessi I, Bilcke J, et al. Detection of human papillomavirus DNA in urine. A review of the literature. Eur J Clin Microbiol Infect Dis. 2012;31(5):627-640.

7. Qiagen.com. Available from: http://www.qiagen.com/resources/ resourcedetail?id=ded1fc02-4a2e-4e6e-9c27-1e0519605b5d\&lang=en. Accessed July 7, 2014. 


\section{Author's response}

\section{Roberta Zappacosta' \\ Giuseppe Lattanzio² \\ Patrizia Viola ${ }^{2}$ \\ Manuel Maria lanieri ${ }^{3}$ \\ Daniela Maria Pia Gatta' \\ Sandra Rosini'}

'Cytopathology Unit, Experimental and Clinical Sciences Department, Gabriele d'Annunzio University of Chieti-Pescara, Chieti, Italy; ${ }^{2}$ Surgical Pathology Unit, ${ }^{3}$ Obstetrics and Gynecology Unit, SS Annunziata Hospital, Chieti, Italy

Correspondence: Roberta Zappacosta

Cytopathology Unit, Experimental and Clinical Sciences Department,

Gabriele d'Annunzio, University of Chieti-Pescara, Via dei Vestini, 66100

Chieti, Italy

Tel +39871357403

Fax +3987I 540079

Email zappacosta2@hotmail.com

Lieveld et al listed six weaknesses of our case report. In this response we will answer their comments point-by-point.

Firstly, they stated that:

Genotype results for lymph nodes and urinary cells are not presented while these results underline the potential role of human papillomavirus (HPV)-53 in oncogenesis.

In the "Results" section we wrote:

...the metastatic lymph nodes showed HPV-53; no HPV was found in the non-metastatic ones. Atypical keratinizing urinary cells also demonstrated a HPV-53 positive result.

Originally, to be concise as per the type of the article (case report), we chose to include only one Figure in our paper relative to reverse transcription polymerase chain reaction (RT-PCR) genotyping results (Figure 3). For the reader's convenience and to further support our findings, here we report the modified Figure 3 as a panel of pictures including the genotyping results for both lymph nodes and atypical urinary cells.

Secondly, referring to our study and in contradiction with the previous comment, Lieveld et al also affirmed that:

It is suggested that HPV-53 is present exclusively in the cervical cancer cells, lymph node metastasis and atypical urinary cells of one single case while the surrounding cervical intraepithelial neoplasia grade 2-or-worse (CIN2+) tissue revealed ten different HPV strains.

Within our "Results" section, we clearly stated that "the CIN2+ tissue near to the invasive cancer showed HPV-16,
$-35,-39,-40,-53,-54,-59,-61,-68$, and $-82 \%$. HPV-53 is listed among the HPV types which have been found in the CIN2+ lesion. On the other hand, HPV-53 alone was detected in the cervical cancer cells, lymph node metastasis, and urinary atypical cells. Such finding supports its causative role during the last phases of cervical oncogenesis.

Thirdly, Lieveld et al wrote that:

It is generally accepted that one lesion is caused by one HPV infection and detection of multiple HPV types thus indicates the presence of multiple infections suggesting that this patient may have several lesions.

They also cited Quint et al to support their assertion. ${ }^{2}$ In the "Introduction" section of their paper, ${ }^{2}$ they clearly confirm the well-known theory according to which:

Multiple HPV genotypes are detected in $15 \%-40 \%$

of women with prevalent CIN of all grades, and in $60 \%$ of women with early incident CIN2 and CIN3.

Moreover, they state that "in approximately $95 \%$ of HPVpositive invasive cervical cancers, only one HPV type is found". These assumptions perfectly fit with the concept that a single clone of dysplastic cervical would progress toward invasive cancer, thus adhering to our findings.

To confirm the polyclonal nature of the CIN and the monoclonal character of the invasive neoplasia, Quint et al define high-grade cervical intraepithelial lesions as "architecturally complex lesions", which are composed by multiple grades of CINs and are "associated with multiple HPV infections". They also conclude that "it is very unlikely that multiple HPV types will be present in one HPV-infected cell". To isolate the single area within high-grade CIN, they utilize laser capture micro-dissection (LCM). Through HPV PCR genotyping performed on LCM-PCR, they demonstrated that there is a single HPV type in $93 \%$ of the cases, and multiple infections in the remaining 7\%. Considering such results, cervical carcinogenesis would be considered as a continuum of events, starting from oncogenic HPV infection(s), proceeding through intraepithelial neoplasia, and reflecting HPV life cycle within the cervical epithelium. At the end of this process, the disruption of $\mathrm{p} 53$ and $\mathrm{pRb}$ mechanisms by persistent HPV infection would favor only one dysplastic clone, maintained by one single HPV type that would be able to switch toward invasive cancer.

The above mentioned theories are widely accepted by the vast majority of the experts in the field and by us. Moreover, it is widely accepted that the overall histological diagnosis is based on the highest grade of CIN detected, 

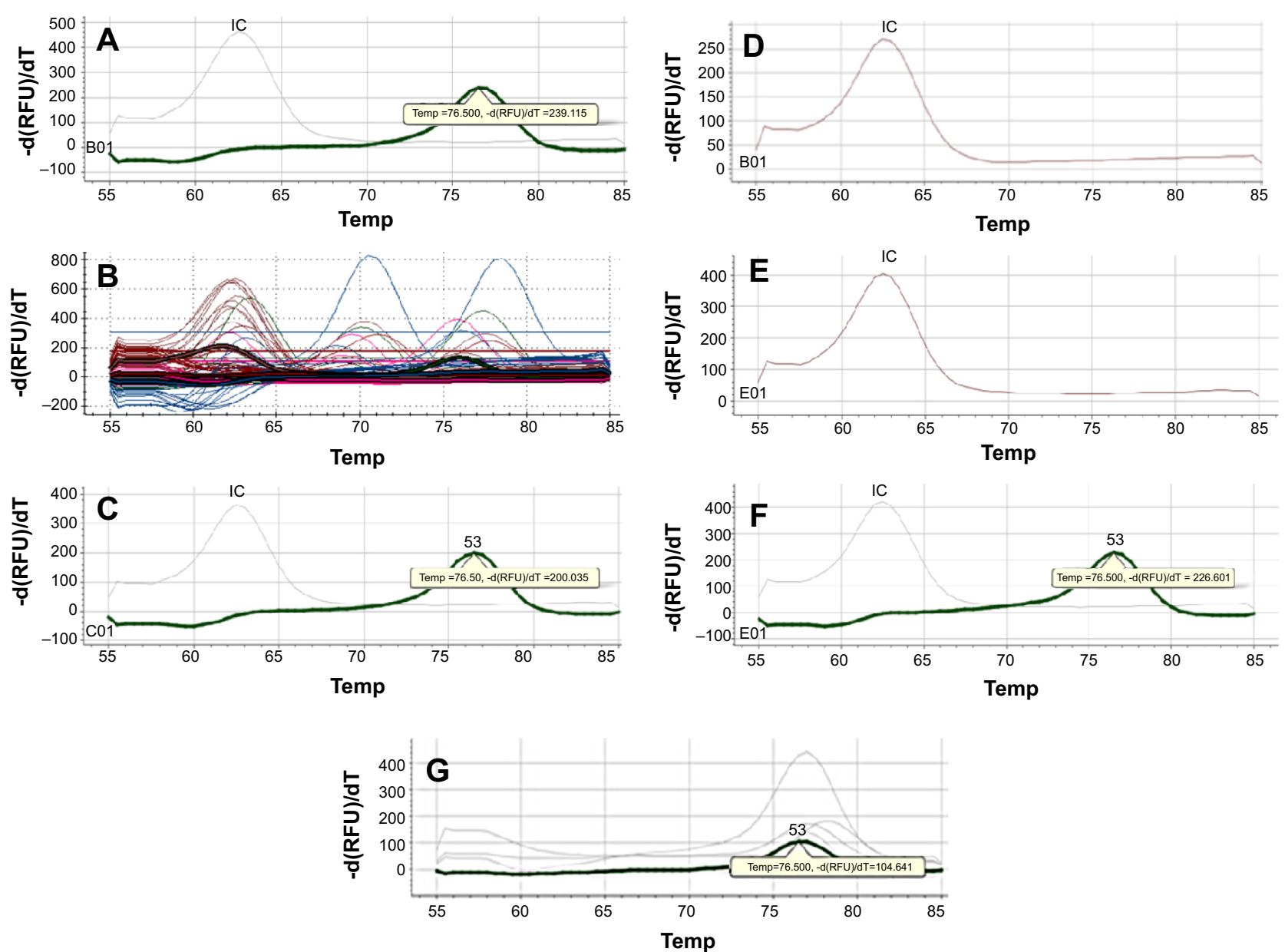

Modified Figure 3 Real-time PCR assay performed on tissues (invasive cervical cancer, CIN2+ lesion adjacent to invasive neoplasia, metastatic lymph nodes, non-metastatic lymph nodes, and thyroid), and on liquid-based urinary samples.

Notes: Anyplex II HPV28 detection assay simultaneously detects 28 HPV genotypes. $\beta$-globin gene is used as a positive internal control to identify processed specimens containing substances that may interfere with PCR amplification. Thyroid tissue was used as an external HPV-negative control. (A) Invasive cervical cancer showing positive result for HPV-53. (B) CIN2+ lesion, in which positive results for HPVs I6, 35, 39, 40, 53, 54, 59, 6I, 68 and 82 have been found. (C) Metastatic lymph nodes, showing HPV-53 positive result. (D) Non-metastatic lymph nodes, testing as HPV-negative. (E) Thyroid tissue testing as HPV-negative. (F) Urinary samples, in which HPV-53 has been found. (G) Melting curve of HPV-53 control included in the PC3 mix of set B, demonstrating the melting curve shape and Tm. The melting profile of samples in which HPV-53 has been found was shown to have a $\mathrm{Tm}$ of $76.5^{\circ} \mathrm{C}$.

Abbreviations: PCR, polymerase chain reaction; $\mathrm{CIN} 2+$, cervical intraepithelial neoplasia grade 2-or-worse; - $d(\mathrm{RFU} / \mathrm{dT})$, change in rate of fluorescence units against temperature; IC, internal control; PC3 of set B, positive control of set B, consisting of a mix of positive controls, including those of HPVs 73, 53, 70, 6, and II; Tm, melting temperature; HPV, human papillomavirus.

since biopsies might also include areas of lower-grade CIN and normal cervical tissue. To perform DNA extraction and RT-genotyping of CIN2+ lesions, we isolated the area corresponding formalin-fixed paraffin embedded (FFPE) immediately adjacent to the invasive cancer using Tru-Cut biopsy needle. The same methods have been used to isolate the area showing infiltration of the basal membrane and stroma. In this way, we demonstrated multiple concurrent genotypes within high-grade CIN (CIN2+), as well as the presence of HPV-53 alone within the infiltrating carcinoma (showing HPV-53 only). We did not use LCM nor HPV LCM-PCR genotyping. Beca et al detected concurrent multiple HPV genotypes in about $37 \%$ of patients with high-grade cervical lesions, and concluded that:
This information might help to better understand the impacts of multiple concurrent genotypes infection in the carcinogenic process and help to set a baseline measure in future studies on the influence of a population-based HPV vaccination. ${ }^{3}$

Based on this adjunctive information, we confirm that our data agrees with previous studies. To further endorse our data, we would like to conclude by reporting the findings from Halec et al. ${ }^{4}$ They demonstrate that when HPV-53 is presented in cervical cancer cells as a single biologically active infection (which is currently considered as a possible high-risk oncogenic HPV by World Health Organization), it would affect the same cellular pathway as any of the fullyrecognized carcinogenic high-risk types. 
Fourthly, Lieveld et al illustrated their disagreement with our results in Figure 3. We would like to apologize since there was a mistake during the editing of this Figure and its legend. We made an error when we were including curves relative to $\mathrm{CIN} 2+$ genotyping rather than to invasive neoplasia, and indicating them as belonging to invasive cancer tissue. We agree that the internal positive control used for Anyplex II HPV28 assay is the human $\beta$-globin, and that a mixture of pathogen clones are used as external positive control (PC) to test the accuracy of master mix in detecting the indicated HPV types. Particularly, positive control for HPV-53 is included in the PC 3 mix of set B. We also understand that HPV-53 type specific pitcher hybridized with the complementary catcher, the last labeled with HEX fluorophore. To avoid any confusion, we would like to make it clear that synthesized HPV-53 plasmid was not used as an extra internal positive control.

Moreover, we agree that a specific melting profile showing a specific melting temperature $(\mathrm{Tm})$ has to be obtained from all samples in which HPV-53 has been found. In this context, we would like to thank Lieveld et al for finding this error. We have decided to publish a corrigendum, in which we included an exhaustive figure-panel representing genotyping results as well as the corrected and more detailed legend. As shown in the modified Figure 3, the melting profile of each sample in which HPV-53 has been detected showed a specific melting curve of HPV-53 with a $\mathrm{Tm}$ of $76.5^{\circ} \mathrm{C}$.

Fifthly, Lieveld et al disagreed that voided urinary samples were firstly analyzed by Hybrid Capture II HPV test (HC2), since to their knowledge HC2 HPV test had not been optimized for urine samples. Numerous studies have been performed to optimize HC2 HPV on urine as stated by Vorsters et al. ${ }^{5}$ In this review, Vorsters conclude that "HPV detection in urine is a feasible practice and a useful tool in future research, but further optimization and standardization is required" and that "different settings may require different methodologies". ${ }^{5}$ It is well known that before introducing a new technology into routinely clinical practice, it should be evaluated under highly controlled experimental conditions.

Sellorf et al analyzed vaginal, vulvar and urine samples of 200 participants for HPV detection. ${ }^{6}$ All these specimens were sent to Digene Corp. Laboratory (now Qiagen) to perform this test. HPVs by HC2 have been detected on 69 urinary specimens. ${ }^{6}$ Percentage agreement between cervical specimens and urine samples for the presence of HPV was found to be $55.2 \%$. Jacobson et al evaluated HPV prevalence in both cervical and urine samples among adolescents by HC2. ${ }^{7}$ Brinkman et al detected HPV by HC2 in $28 \%$ of urine specimens of HIV-positive women. ${ }^{8}$ Martin-Ezquerra et al estimated the incidence of HPV infection in urinary samples of 91 male sexual partners of women diagnosed with CIN2-3. ${ }^{9}$ They concluded that "HC2 is an easy and economical method whose usefulness has been previously demonstrated". 9

In our setting HC2 technology is routinely used to manage women reporting low grade squamous intraepithelial lesion diagnosis, and during follow-ups after treatment for $\mathrm{CIN}$. To evaluate diagnostic performance of $\mathrm{HC} 2$ on urinary specimens and to contribute to further research in the field, this test has to be evaluated in clinical practice. In our paper, when we stated that "all the cervical cytological specimens resulted as high-risk HPV-positive", we implied that atypical urinary cells were shown to be HC2 negative since HPV-53 is not included within the 13 oncogenic genotypes included in $\mathrm{HC} 2$ probe detected by $\mathrm{HC} 2$ probe.

Finally, Lieveld et al asked for additional details regarding the laboratory measures employed to prevent cross-contamination. In our setting, microtome blade and the working space were rinsed with DNA Away ${ }^{\mathrm{TM}}$ before we handled each tissue block. Before sectioning negative control FFPE (human thyroid, previously tested for HPV infection), we also changed microtome blade. The melting curve related to negative control is shown in Figure 3E. We have omitted much of our laboratory measures in our paper as it was a case report rather than an original research paper.

In summary, apologizing again for our mistake made in the original Figure 3, here we confirm the causative role of HPV-53 in the invasive cervical cancer in our patient.

\section{Disclosure}

The authors report no conflicts of interest in this work.

\section{References}

1. Zappacosta R, Lattanzio G, Viola P, Ianieri MM, Gatta DMP, Rosini S A very rare case of HPV-53-related cervical cancer, in a 79-year-old woman with a previous history of negative Pap cytology. Clin Interv Aging. 2014;9:683-688.

2. Quint W, Jenkins D, Molijin A, et al. One virus, one lesion-individual components of CIN lesions contain a specific HPV types. J Pathol. 2012; 227(1):62-71.

3. Beca F, Pinheiro J, Rios E, Pontes P, Amendoeira I. Genotypes and Prevalence of HPV Single and Multiple Concurrent Infections in Women with HSIL. Diagn Cytopathol. Epub 2014 Mar 13.

4. Halec G, Alemany A, LIoveras B, et al. Pathogenic role of the eight probably/possibly carcinogenic HPV types 26, 53, 66, 67, 68, 70, 73 and 82 in cervical cancer. J Pathol. Epub 2014 Jul 16.

5. Vorsters A, Micalessi I, Bilcke J, et al. Detection of human papillomavirus DNA in urine. A review of the literature. Eur J Clin Microbiol Infect Dis. 2012;31(5):627-640.

6. Sellorf J, Lorincz AT, Mahony JB, et al. Comparison of self-collected vaginal, vulvar and urine sampes with physician-collected cervical samples for human papillomavirus testing to detect high-grade squamous intraepithelial lesions. JAMC. 2000;163(5):513-518. 
7. Jacobson DL, Womack SD, Peralta L. et al. Concordance of human papillomavirus in the cervix and urine among inner city adolescents. Pediatr Infect Dis J. 2000;19(8):722-728.

8. Brinkman JA, Jones WE, Gaffda A, et al. Detection of human papillomavirus DNA in urine specimens from human immunodeficiency virus-positive women. JCM. 2002;40(9):3155-3161.
9. Martin-Ezquerra G, Fuste P, Larrazabal F, et al. Incidence of human Papillomavirus infection in male sexual partners of women diagnosed with CIN II-III. Eur J Dermatol. 2012;22(2):200-204.

\section{Publish your work in this journal}

Clinical Interventions in Aging is an international, peer-reviewed journal focusing on evidence-based reports on the value or lack thereof of treatments intended to prevent or delay the onset of maladaptive correlates of aging in human beings. This journal is indexed on PubMed Central, MedLine,
CAS, Scopus and the Elsevier Bibliographic databases. The manuscript management system is completely online and includes a very quick and fair peer-review system, which is all easy to use. Visit http://www.dovepress. com/testimonials.php to read real quotes from published authors. 